\title{
TRANSFER AND A SUPREMUM PRINCIPLE FOR ERNA
}

\author{
CHRIS IMPENS AND SAM SANDERS
}

\begin{abstract}
Elementary Recursive Nonstandard Analysis, in short ERNA, is a constructive system of nonstandard analysis proposed around 1995 by Patrick Suppes and Richard Sommer, who also proved its consistency inside PRA. It is based on an earlier system developed by Rolando Chuaqui and Patrick Suppes, of which Michal Rössler and Emil Jeřábek have recently proposed a weakened version. We add a $\Pi_{1}$-transfer principle to ERNA and prove the consistency of the extended theory inside PRA. In this extension of ERNA a $\Sigma_{1}$-supremum principle 'up-to-infinitesimals', and some well-known calculus results for sequences are deduced. Finally, we prove that transfer is 'too strong' for finitism by reconsidering Rössler and Jeřábek's conclusions.
\end{abstract}

§1. Introduction. Hilbert's Program, proposed in 1921, called for an axiomatic formalization of mathematics, together with a proof that this axiomatization is consistent. The consistency proof itself was to be carried out using only what Hilbert called finitary methods. In due time, many characterized Hilbert's informal notion of 'finitary' as that which can be formalized in Primitive Recursive Arithmetic (PRA), proposed in 1923 by Skolem.

By Gödel's second incompleteness theorem (1931) it became evident that only partial realizations of Hilbert's program are possible. The system proposed by Chuaqui and Suppes, recently adapted by Rössler and Jeřábek, is such a partial realization, in that it provides an axiomatic foundation for basic analysis, with a PRA consistency proof $[2,9]$. Sommer and Suppes's improved system allows definition by recursion, which does away with a lot of explicit axioms, and still has a PRA proof of consistency [10, p. 21]. This system is called Elementary Recursive Nonstandard Analysis, in short ERNA. Its consistency is proved via Herbrand's Theorem (1930), which is restricted to quantifier-free formulas $Q\left(x_{1}, \ldots, x_{n}\right)$, usually containing free variables. Alternatively, one might say it is restricted to universal sentences

$$
\left(\forall x_{1}\right) \ldots\left(\forall x_{n}\right) Q\left(x_{1}, \ldots, x_{n}\right) .
$$

We will use Herbrand's theorem in the following form (see [2] and [10]); for more details, see [1] and [3].

1. THEOREM. A quantifier-free theory $T$ is consistent if and only if every finite set of instantiated axioms of $T$ is consistent.

Instantiating a formula $\varphi\left(x_{1}, \ldots, x_{k}\right)$ means that every occurrence of a free variable $x_{i}$ is replaced with a constant $\tau_{i}$. Since Herbrand's theorem requires that

Received April 30, 2007. 
ERNA's axioms be written in a quantifier-free form, some axioms definitely look artificial. Fortunately, theorems don't suffer from the quantifier-free restriction.

§2. ERNA, the system. In this section we describe ERNA and its fundamental features. Undocumented results are quoted from [10].

2. Notation. $\mathbb{N}=\{0,1,2, \ldots\}$ consists of the (finite) nonnegative integers.

3. Notation. $\vec{x}$ stands for some finite (possibly empty) sequence $\left(x_{1}, \ldots, x_{k}\right)$.

4. Notation. $\tau(\vec{x})$ denotes a term in which $\vec{x}=\left(x_{1}, \ldots, x_{k}\right)$ is the list of the distinct free variables.

\subsection{The language.}

- connectives: $\wedge, \neg, \vee, \rightarrow, \leftrightarrow$,

- quantifiers: $\forall, \exists$,

- an infinite set of variables,

- relation symbols: ${ }^{1}$

- binary $x=y$,

- binary $x \leq y$,

- unary $\mathscr{F}(x)$, read as ' $x$ is infinitesimal', also written ' $x \approx 0$ ',

- unary $\mathscr{N}(x)$, read as ' $x$ is hypernatural',

- individual constant symbols:

-0 ,

-1 ,

- $\varepsilon$ (axiom 11.(6) asserts that $\varepsilon$ is a positive infinitesimal hyperrational),

- $\omega$ (the axioms 11.(7) and 7.(4) assert that $\omega=1 / \varepsilon$ is an infinite hypernatural),

- $\uparrow$, to be read as 'undefined'.

5. Notation. ' $x$ is defined' stands for ' $x \neq \uparrow$ '. (Example: $1 / 0$ is undefined, $1 / 0=\uparrow$.)

- function symbols: ${ }^{2}$

- (unary) 'absolute value' $|x|$, 'ceiling' $\lceil x\rceil$, 'weight' $\|x\|$. (For the meaning of $\|x\|$, see Theorem 23.)

- (binary) $x+y, x-y, x . y, x / y, x^{\wedge} y$. (Axiom set 18 and Axiom 39.(4) assert that $x^{\wedge} n=x^{n}$ for hypernatural $n$, else undefined.)

- for each $k \in \mathbb{N}, k k$-ary function symbols $\pi_{k, i}(i=1, \ldots, k)$. (Axiom schema 19 asserts that $\pi_{k, i}(\vec{x})$ are the projections of the $k$-tuple $\vec{x}$.)

- for each quantifier-free formula $\varphi$ with $m+1$ free variables, not involving min, an $m$-ary function symbol $\min _{\varphi}$. (For the meaning of which, see Theorems 32 and 38.)

- for each triple $\left(k, \sigma\left(x_{1}, \ldots, x_{m}\right), \tau\left(x_{1}, \ldots, x_{m+2}\right)\right)$ with $0<k \in \mathbb{N}, \sigma$ and $\tau$ terms not involving min, an $(m+1)$-ary function symbol rec $\sigma_{\sigma \tau}^{k}$. (Axiom schema 28 asserts that this is the term obtained from $\sigma$ and $\tau$ by recursion, after the model $f(0, \vec{x})=\sigma(\vec{x}), f(n+1, \vec{x})=\tau(f(n, \vec{x}), n, \vec{x})$, if terms are defined and 'do not weigh too much'.)

\footnotetext{
${ }^{1}$ For better readibility we express unary relations in $x$ and binary ones in $(x, y)$.

${ }^{2}$ We denote the values as computed in $x$ or $(x, y)$ according to the arity.
} 


\subsection{The axioms.}

6. AхIOM SET (Logic). Axioms of first-order logic.

7. АхіOм SET (Hypernaturals).

(1) 0 is hypernatural,

(2) if $x$ is hypernatural, so is $x+1$,

(3) if $x$ is hypernatural, then $x \geq 0$,

(4) $\omega$ is hypernatural.

8. Definition. ' $x$ is infinite' stands for ' $x \neq 0 \wedge 1 / x \approx 0$ '; ' $x$ is finite' stands for ' $x$ is not infinite'; ' $x$ is natural' stands for ' $x$ is hypernatural and finite'.

9. Definition. A term or formula is called internal if it does not involve $\mathscr{I}$; if it does, it is called external.

10. Notation. The variables $n, m, k, l, \ldots$, both lower and upper-case, will represent hypernatural variables.

11. AхIOM SET (Infinitesimals).

(1) if $x$ and $y$ are infinitesimal, so is $x+y$,

(2) if $x$ is infinitesimal and $y$ is finite, $x y$ is infinitesimal,

(3) an infinitesimal is finite,

(4) if $x$ is infinitesimal and $|y| \leq x$, then $y$ is infinitesimal,

(5) if $x$ and $y$ are finite, so is $x+y$,

(6) $\varepsilon$ is infinitesimal,

(7) $\varepsilon=1 / \omega$.

12. COROLlary. 1 is finite.

Proof. If 1 is infinite, its inverse is infinitesimal, i.e., $1 \approx 0$. By axiom 11.(3), it would follow that 1 is finite, contradicting the assumption.

13. AхIOM SET (Ordered field). Axioms expressing that ERNA's defined elements constitute an ordered field of characteristic zero with an absolute-value function. These quantifier-free axioms include

- if $x$ is defined, then $x+0=0+x=x$,

- if $x$ is defined, then $x+(0-x)=(0-x)+x=0$,

- if $x$ is defined and $x \neq 0$, then $x .(1 / x)=(1 / x) \cdot x=1$.

14. Ахіом (Archimedean). If $x$ is defined, $|\lceil x\rceil|$ is a hypernatural and $\lceil x\rceil-1<$ $x \leq\lceil x\rceil$.

15. THEOREM. If $x$ is defined, then $\lceil x\rceil$ is the least integer $\geq x$.

16. THEOREM. $x$ is finite iff there is a natural $n$ such that $|x| \leq n$.

PRoof. The statement is trivial for $x=0$. If $x \neq 0$ is finite, so is $|x|$ because, assuming the opposite, $1 /|x|$ would be infinitesimal and so would $1 / x$ be by axiom 11.(4). By axiom 11.(5), the hypernatural $n=\lceil|x|\rceil<|x|+1$ is then also finite. Conversely, let $n$ be natural and $|x| \leq n$. If $1 /|x|$ were infinitesimal, so would $1 / n$ be by axiom 11.(4), and this contradicts the assumption that $n$ is finite.

17. Corollary. $x \approx 0$ iff $|x|<1 / n$ for all natural $n \geq 1$.

18. AXIOM SET (Power).

(1) if $x$ is defined, then $x^{\wedge} 0=1$,

(2) if $x$ is defined and $n$ is hypernatural, then $x^{\wedge}(n+1)=\left(x^{\wedge} n\right) x$. 
19. Axiom Schema (Projection). If $x_{1}, \ldots, x_{n}$ are defined, then $\pi_{n, i}\left(x_{1}, \ldots, x_{n}\right)=$ $x_{i}$ for $i=1, \ldots, n$.

20. Axiom SET (Weight).

(1) if $\|x\|$ is defined, then $\|x\|$ is a nonzero hypernatural,

(2) if $|x|=m / n \leq 1$ ( $m$ and $n \neq 0$ hypernaturals), then $\|x\|$ is defined, $\|x\| .|x|$ is hypernatural and $\|x\| \leq n$,

(3) if $|x|=m / n \geq 1$ ( $m$ and $n \neq 0$ hypernaturals), then $\|x\|$ is defined, $\|x\| /|x|$ is hypernatural and $\|x\| \leq m$.

21. Definition. A (hyper)rational is of the form $\pm p / q$, with $p$ and $q \neq 0$ (hyper)natural. We also use 'standard' instead of 'rational'.

22. Notation. $\left(\forall^{s t} x\right) \varphi(x)$ stands for $(\forall x)(x$ is standard $\rightarrow \varphi(x))$ and $\left(\exists^{s t} x\right) \varphi(x)$ for $(\exists x)(x$ is standard $\wedge \varphi(x))$.

\section{THEOREM.}

(1) If $x$ is not a hyperrational, then $\|x\|$ is undefined.

(2) If $x= \pm p / q$ with $p$ and $q \neq 0$ relatively prime hypernaturals, then

$$
\| \pm p / q\|=\max \{|p|,|q|\} \text {. }
$$

24. THEOREM.

(1) $\|0\|=1$,

(2) if $n \geq 1$ is hypernatural, $\|n\|=n$,

(3) if $\|x\|$ is defined, then $\|1 / x\|=\|x\|$ and $\|\lceil x\rceil\| \leq\|x\|$,

(4) if $\|x\|$ and $\|y\|$ are defined, $\|x+y\|,\|x-y\|,\|x y\|$ and $\|x / y\|$ are at most equal to $(1+\|x\|)(1+\|y\|)$, and $\left\|x^{\wedge} y\right\|$ is at most $(1+\|x\|)^{\wedge}(1+\|y\|)$.

25. Notation. For any $0<n \in \mathbb{N}$ we write

$$
\left\|\left(x_{1}, \ldots, x_{n}\right)\right\|=\max \left\{\left\|x_{1}\right\|, \ldots,\left\|x_{n}\right\|\right\} .
$$

26. Notation. For any $0<n \in \mathbb{N}$ we write

$$
2_{n}^{x}:=\underbrace{2^{\wedge}\left(\cdots 2^{\wedge}\left(2^{\wedge}\left(2^{\wedge} x\right)\right)\right)}_{n \text { 2`s }} .
$$

27. THEOREM. If the term $\tau(\vec{x})$ is defined and does not involve $\omega$, rec or min, then there exists $a 0<k \in \mathbb{N}$ such that

$$
\|\tau(\vec{x})\| \leq 2_{k}^{\|\vec{x}\|}
$$

28. Aхіом schema (Recursion). For any $0<k \in \mathbb{N}$ and internal $\sigma$, $\tau$ not involving $\min :$

$$
\begin{gathered}
\operatorname{rec}_{\sigma \tau}^{k}(0, \vec{x})= \begin{cases}\sigma(\vec{x}) & \text { if this is defined, and has weight } \leq 22_{k}^{\|\vec{x}\|}, \\
\uparrow & \text { if } \sigma(\vec{x})=\uparrow, \\
0 & \text { otherwise. }\end{cases} \\
\operatorname{rec}_{\sigma \tau}^{k}(n+1, \vec{x})= \begin{cases}\tau\left(\operatorname{rec}_{\sigma \tau}^{k}(n, \vec{x}), n, \vec{x}\right) & \text { if defined, with weight } \leq 22_{k}^{\|\vec{x}, n+1\|}, \\
\uparrow & \text { if } \tau\left(\operatorname{rec}_{\sigma \tau}^{k}(n, \vec{x}), n, \vec{x}\right)=\uparrow, \\
0 & \text { otherwise. }\end{cases}
\end{gathered}
$$


If the list $\vec{x}$ is empty, the above reduces to

$$
\begin{gathered}
\operatorname{rec}_{\sigma \tau}^{k}(0)=\sigma, \\
\operatorname{rec}_{\sigma \tau}^{k}(n+1)= \begin{cases}\tau\left(\operatorname{rec}_{\sigma \tau}^{k}(n), n\right) & \text { if defined, with weight } \leq 2_{k}^{n+1}, \\
\uparrow & \text { if } \tau\left(\operatorname{rec}_{\sigma \tau}^{k}(n), n\right)=\uparrow, \\
0 & \text { otherwise. }\end{cases}
\end{gathered}
$$

29. Corollary. If $\operatorname{rec}_{\sigma \tau}^{k}(n, \vec{x})$ is defined, then $\left\|\operatorname{rec}_{\sigma \tau}^{k}(n, \vec{x})\right\| \leq 2_{k}^{\|\vec{x}, n\|}$.

We now adapt theorem 27 so as to allow more general terms.

30. THEOREM.

(1) If the term $\tau_{1}(\vec{x})$ is defined and does not involve $\omega$ or min, then there exists a $0<k \in \mathbb{N}$ such that $\left\|\tau_{1}(\vec{x})\right\| \leq 2_{k}^{\|\vec{x}\|}$.

(2) If the term $\tau_{2}(\vec{x})$ is defined and does not involve min, then there exists a $0<k \in \mathbb{N}$ such that $\left\|\tau_{2}(\vec{x})\right\| \leq 2_{k}^{\|\vec{x}, \omega\|}$.

Proof. For $(1)$, we replace in $\tau_{1}(\vec{x})$ every term $\operatorname{rec}_{\sigma \tau}^{k}(n, \vec{y})$ by the corresponding term $2_{k}^{\|\vec{y}, n\|}$. For the resulting term $\tau_{1}^{\prime}(\vec{x})$ we have $\left\|\tau_{1}(\vec{x})\right\| \leq\left\|\tau_{1}^{\prime}(\vec{x})\right\|$ by the preceding corollary. As the new term is defined and does not involve $\omega$, min or rec, theorem 27 implies there is a $0<k \in \mathbb{N}$ such that $\left\|\tau_{1}^{\prime}(\vec{x})\right\| \leq 2_{k}^{\|\vec{x}\|}$. For (2), let $\tau_{2}^{\prime}(\vec{x}, m)$ be the term obtained by replacing, in $\tau_{2}(\vec{x})$, every occurrence of $\omega$ by $m$, and every occurrence of $\varepsilon$ by $1 / m$. By the previous item, there is a $0<k \in \mathbb{N}$ such that $\left\|\tau_{2}^{\prime}(\vec{x}, m)\right\| \leq 2_{k}^{\|\vec{x}, m\|}$. Hence $\left\|\tau_{2}(\vec{x})\right\|=\left\|\tau_{2}^{\prime}(\vec{x}, \omega)\right\| \leq 2_{k}^{\|\vec{x}, \omega\|}$.

31. Axiom SCHEMA (Internal minimum). For every internal quantifier-free formula $\varphi(y, \vec{x})$ not involving $\min$ we have

(1) $\min _{\varphi}(\vec{x})$ is a hypernatural number,

(2) if $\min _{\varphi}(\vec{x})>0$, then $\varphi\left(\min _{\varphi}(\vec{x}), \vec{x}\right)$,

(3) if $n$ is a hypernatural and $\varphi(n, \vec{x})$, then $\min _{\varphi}(\vec{x}) \leq n$ and $\varphi\left(\min _{\varphi}(\vec{x}), \vec{x}\right)$.

32. THEOREM. If the internal quantifier-free formula $\varphi(y, \vec{x})$ does not involve min, and if there are hypernatural n's such that $\varphi(n, \vec{x})$, then $\min _{\varphi}(\vec{x})$ is the least of these. If there are none, $\min _{\varphi}(\vec{x})=0$.

33. THEOREM (Hypernatural induction). Let $\varphi(n)$ be an internal quantifier-free formula not involving $\mathrm{min}$, such that

(1) $\varphi(0)$,

(2) $\varphi(n) \rightarrow \varphi(n+1)$.

Then $\varphi(n)$ holds for all hypernatural $n$.

Proof. Suppose, on the contrary, that there is a hypernatural $n$ such that $\neg \varphi(n)$. By Theorem 32, there is a least hypernatural $n_{0}$ such that $\neg \varphi\left(n_{0}\right)$. By our assumption (1), $n_{0}>0$. Consequently, $\varphi\left(n_{0}-1\right)$ does hold. But then, by our assumption (2), so would $\varphi\left(n_{0}\right)$. This contradiction proves the theorem.

34. EXAMPLE. If $f(n)$ is an internal function not involving min and such that $0<f(n) \leq \omega$ for all $n$, then $0<\operatorname{rec}_{0 f}^{1}(n) \leq \omega$ for all $n>0$.

A more important application is hypernatural overflow and underflow in ERNA.

35. THEOREM. Let $\varphi(n)$ be an internal quantifier-free formula, not involving $\mathrm{min}$. 
(1) If $\varphi(n)$ holds for every natural $n$, it holds for all hypernatural $n$ up to some infinite hypernatural $\bar{n}$ (overflow).

(2) If $\varphi(n)$ holds for every infinite hypernatural $n$, it holds for all hypernatural $n$ from some natural $\underline{n}$ on (underflow).

PRoof. If $\varphi(n)$ holds for every hypernatural $n$, any $\bar{n}$ and $\underline{n}$ will do. If not, $n_{0}=\min _{\neg \varphi}$ is the least hypernatural for which $\varphi$ does not hold, and $n_{1}=\min _{\neg \varphi^{\prime}}$ is the least hypernatural for which $\varphi^{\prime}(n):=\varphi(\omega-n)$ does not hold. By the assumption in (1), $n_{0}$ is infinite, and it follows that $\varphi(n)$ holds for every hypernatural $n \leq \bar{n}:=n_{0}-1$. By the assumption in (2), $\omega-n_{1}$ is finite, and so is $\underline{n}:=\omega-n_{1}+1$. For $\underline{n} \leq n \leq \omega$ we have $0 \leq \omega-n \leq n_{1}-1$, implying that $\varphi^{\prime}(\omega-n)=\varphi(n)$ holds. For $n>\omega, \varphi(n)$ holds by assumption. Hence $\underline{n}$ satisfies the requirements.

In (12) and (13) we give explicit formulas for $\bar{n}$ and $\underline{n}$. This theorem allows us to prove Robinson's sequential lemma, (see [11, p. 150]), in ERNA.

36. Corollary. Let $f(n)$ be an internal function not involving min. If $f(n) \approx 0$ for all $n \in \mathbb{N}$, then $f(n) \approx 0$ for all hypernatural $n$ up to some infinite hypernatural $\omega_{1}$.

Proof. Apply overflow to the formula $|f(n)|<1 / n$.

37. Aхіом sChema (External minimum). For every (possibly external) quantifierfree formula $\varphi(y, \vec{x})$ not involving $\min$ or $\omega$ we have

(1) $\min _{\varphi}(\vec{x})$ is a hypernatural number,

(2) if $\min _{\varphi}(\vec{x})>0$, then $\varphi\left(\min _{\varphi}(\vec{x}), \vec{x}\right)$,

(3) if $n$ is a natural number, $\|\vec{x}\|$ is finite and $\varphi(n, \vec{x})$, then $\min _{\varphi}(\vec{x}) \leq n$ and $\varphi\left(\min _{\varphi}(\vec{x}), \vec{x}\right)$.

38. THEOREM. Let $\varphi(n, \vec{x})$ be a (possibly external) quantifier-free formula not involving min or $\omega$. If $\|\vec{x}\|$ is finite and if there are natural n's such that $\varphi(n, \vec{x})$, then $\min _{\varphi}(\vec{x})$ is the least of these. If there are none, $\min _{\varphi}(\vec{x})=0$.

This theorem can be used to produce proofs by natural induction.

39. Ахіом SET ((Un)defined terms).

(1) $0,1, \omega, \varepsilon$ are defined,

(2) $|x|,\lceil x\rceil,\|x\|$ are defined iff $x$ is,

(3) $x+y, x-y, x y$ are defined iff $x$ and $y$ are; $x / y$ is defined iff $x$ and $y$ are and $y \neq 0$,

(4) $x^{\wedge} y$ is defined iff $x$ and $y$ are and $y$ is hypernatural,

(5) $\pi_{k, i}\left(x_{1}, \ldots, x_{k}\right)$ is defined iff $x_{1}, \ldots, x_{k}$ are,

(6) if $x$ is not a hypernatural, $\operatorname{rec}_{\sigma \tau}^{k}(x, \vec{y})$ is undefined,

(7) $\min _{\varphi}\left(x_{1}, \ldots, x_{k}\right)$ is defined iff $x_{1}, \ldots, x_{k}$ are.

40. Corollary. In ERNA, 'defined' and 'hyperrational' mean the same.

Proof. Let $x$ be non-hyperrational. From theorem 23 we obtain that $\|x\|$ is undefined, and so is $x$ by item (2) of the last axiom set. Hence $\uparrow$ is the only non-hyperrational element in ERNA.

Note that ERNA has no 'standard part' function st with the property that $\operatorname{st}(\varepsilon)=$ 0 for $\varepsilon \approx 0$, which would allow for the unique decomposition of a finite number as the sum of a standard and an infinitesimal number, sometimes called the 'fundamental theorem of nonstandard analysis', [5]. Indeed, with such a function st, ERNA 
would allow to construct the field of real numbers. As ERNA's consistency is proved in PRA, the latter would also allow to construct the real number field, something which is known to be impossible, [6].

Although the real number field is not available in ERNA, the rationals will turn out to be dense in the finite part of ERNA's field, see theorem 56 .

§3. ERNA+Transfer. In nonstandard mathematics, Transfer expresses Leibniz's principle that the 'same' laws hold for standard and nonstandard objects alike. In this section, we will add such a principle to ERNA and prove the consistency of the extended theory inside PRA.

41. Definition. If $\tau$ is an individual constant, the depth $d(\tau)$ is zero. For a term $\tau\left(x_{1}, \ldots, x_{k}\right)$ we put $d\left(\tau\left(x_{1}, \ldots, x_{k}\right)\right)=\max \left\{d\left(x_{1}\right), \ldots, d\left(x_{k}\right)\right\}+1$.

42. Definition. If $L$ is the language of ERNA, then $L^{\text {st }}$, the standard language of ERNA, is $L$ without $\omega, \varepsilon$ or $\mathscr{I}$.

43. Aхіом SCHEMA ( $\Pi_{1}$-transfer). For every quantifier-free formula $\varphi(n)$ from $L^{s t}$, not involving min, we have

$$
\varphi(n+1) \vee\left(0<\min _{\neg \varphi}=\text { finite }\right) .
$$

We denote by ERNA $+\Pi_{1}$-TRANS the original theory with the preceding axiom schema (1) added. The latter expresses in a quantifier-free way the basic transfer principle $\left(\forall^{s t} n \geq 1\right) \varphi(n) \rightarrow(\forall n \geq 1) \varphi(n)$. After the consistency proof of ERNA $+\Pi_{1}$-TRANS, the reasons for the restrictions on $\varphi$ will become apparent.

Before going into the consistency of ERNA $+\Pi_{1}$-TRANS, let us briefly review the consistency proof of ERNA. In view of Herbrand's theorem, we have to prove that any finite set $T$ of instantiated axioms of ERNA is consistent. This we do by means of a mapping val. It maps all terms in $T$ to functions of rationals and all relations in $T$ to relations between rationals, in such a way that all the axioms in $T$ receive the predicate 'true'. When this is achieved, $T$ has a model.

The construction of val requires $D$ steps, where $D$ is the maximal depth of the finitely many terms occurring in $T$.

Three rational numbers $0<a_{0}<b_{0}<c_{0}$ being chosen, ERNA's terms of zero depth are interpreted as $\operatorname{val}(0)=0, \operatorname{val}(1)=1, \operatorname{val}(\omega)=b_{0}$ and $\operatorname{val}(\varepsilon)=1 / b_{0}$.

After a finite number $D$ of inductive steps, each one allowing terms of greater depth, all terms in $T$ have been interpreted in such a way that $|\operatorname{val}(\tau)|$ belongs to $\left[0, a_{D}\right],\left[b_{D}, c_{D}\right]$ or $\left[1 / c_{D}, 1 / b_{D}\right]$, according to $\tau$ being finite, infinite or infinitesimal. Finally, $\operatorname{val}(x \approx 0)$ is defined by $|\operatorname{val}(x)| \leq 1 / b_{D}$. Thus all of ERNA's relations and terms have been given an interpretation. All that is left, is to check that all axioms in $T$ receive the predicate 'true' under this interpretation. For this rather technical verification we refer to [10].

By theorem 30 there is a $0<B \in \mathbb{N}$ such that for every term (of which there are only finitely many) $f(\vec{x})$ occurring in $T$, not involving min, we have

$$
\|f(\vec{x})\| \leq 2_{B}^{\|\vec{x}\|} .
$$

Note that $\omega$, which is allowed to occur, has been replaced with an extra free variable as in [10]. 
Then define

$$
\begin{gathered}
f_{0}(x)=2_{B}^{x} \text { and } f_{n+1}(x)=f_{n}^{t}(x)=\underbrace{f_{n}\left(f_{n}\left(\ldots\left(f_{n}(x)\right)\right)\right)}_{t f_{n} \text { 's }} \\
a_{0}=1, b_{0}=f_{D+1}\left(a_{0}\right), c_{0}=b_{0}, d_{0}=f_{D+1}\left(c_{0}\right)
\end{gathered}
$$

and

$$
a_{i+1}=f_{D-i}^{j}\left(a_{i}\right), b_{i}=f_{D-i}^{j+1}\left(a_{i}\right), c_{i+1}=f_{D-i}^{l}\left(c_{i}\right), d_{i+1}=f_{D-i}^{l+1}\left(c_{i}\right) .
$$

The numbers $t, j$ and $l$ are determined by the terms in the set $T$, their depths and the bounds on their weight; see [10] for details. Note that if we increase $B$ to $B^{\prime}>B$ and use $f_{0}^{\prime}(x)=2_{B^{\prime}}^{x}$, the same $D$-step process as above would still yield a valid val ${ }^{\prime}$ for $T$. Also, $\operatorname{val}(\varphi(\vec{x}))=\varphi(\operatorname{val}(\vec{x}))$ for every quantifier-free formula $\varphi$ of $L^{\text {st }}$ not involving min; see [10] for details.

44. THEOREM. ERNA $+\Pi_{1}$-TRANS is consistent and this consistency can be proved by a finite iteration of ERNA's consistency proof.

Proof. Let $T$ be any finite set of instantiated axioms of ERNA $+\Pi_{1}$-TRANS. Let $D$ be the maximum depth of the terms in $T$. Let $\varphi_{1}(n), \ldots, \varphi_{N}(n)$ be the quantifier-free formulas from $L^{s t}$ whose $\Pi_{1}$-transfer axiom (1) occurs in $T$. Leaving out these axioms from $T$, we are left with a finite set $T^{\prime}$ of instantiated ERNA axioms. Let val be its interpretation into the rationals as sketched above. If we have

$$
(\forall i \in\{1, \ldots, N\})\left(\left(\exists m \leq a_{D}\right) \neg \varphi_{i}(m) \vee\left(\forall n \in\left[0, a_{D}\right] \cup\left[b_{D}, c_{D}\right]\right) \varphi_{i}(n)\right),
$$

recalling that val maps infinite numbers into $\left[b_{D}, c_{D}\right]$, we see that val provides a true interpretation of the whole of $T$, not just $T^{\prime}$. On the other hand, assume there is an exceptional $\varphi^{\prime}:=\varphi_{i}$ for which

$$
\left(\forall m \leq a_{D}\right) \varphi^{\prime}(m) \wedge\left(\exists n \in\left[0, a_{D}\right] \cup\left[b_{D}, c_{D}\right]\right) \neg \varphi^{\prime}(n) .
$$

Note that this implies $\left(\exists n \in\left[b_{D}, c_{D}\right]\right) \neg \varphi^{\prime}(n)$. Now choose a natural $B^{\prime}>B$ such that $2_{B^{\prime}}^{1}>c_{D}$, redefine $f_{0}(x)$ as $2_{B^{\prime}}^{x}$ and construct an interpretation val' in the same way as before. This val' continues to make the axioms in $T^{\prime}$ true and does the same with the axiom

$$
\varphi^{\prime}(n+1) \vee\left(0<\min _{\neg \varphi^{\prime}}=\text { finite }\right) .
$$

Indeed, if a hypernatural $n$ with $\operatorname{val}(n) \in\left[b_{D}, c_{D}\right]$ makes $\varphi^{\prime}$ false, it is interpreted by val $^{\prime}$ as a finite number because $n \leq c_{D} \leq a_{D}^{\prime}$ by our choice of $B^{\prime}$. Then the sentence $\left(\exists n \leq a_{D}^{\prime}\right) \neg \varphi^{\prime}(n)$ is true; hence, $\left(0<\min _{\neg \varphi^{\prime}}=\right.$ finite $)$ is true under val' and so is the whole of $(5)$.

Define $T^{\prime \prime}$ as $T^{\prime}$ plus all instances of (5) occurring in $T$. If there is another exceptional $\varphi_{i}$ such that (4) holds, repeat this process. Note that if we increase $B^{\prime}$ to $B^{\prime \prime}>B^{\prime}$, redefine $f_{0}(x)$ as $2_{B^{\prime \prime}}^{x}$ and construct val ${ }^{\prime \prime}$, the latter still makes the axioms of $T^{\prime}$ true, but the axioms of $T^{\prime \prime}$ as well, since $a_{D}^{\prime} \leq a_{D}^{\prime \prime}$ and hence (5) is true under val" for the same reason as for val'.

This process, repeated, will certainly halt: either the list $\{1, \ldots, N\}$ becomes exhausted or, at some earlier stage, a valid interpretation is found for $T$. Note that this consistency proof, requiring at most $N D$ steps, is a finite iteration of ERNA's, which requires at most $D$ steps. 
The restrictions on the formulas $\varphi$ admitted in (1) are imposed by our consistency proof. Neither $\approx$ nor $\omega$ can occur, because in ERNA's consistency proof, $\omega$ is interpreted as $b_{0}$ and ' $x \approx 0$ ' as ' $|x| \leq 1 / b_{D}$ ', both of which depend on $B$. By our changing $B$ into $B^{\prime}>B$, formulas like (5) could loose their 'true' interpretation from one step to the next. The exclusion of min has, of course, a different reason: $\min _{\varphi}$ is only allowed in ERNA when $\varphi$ does not rely on min.

Note that Parson's theorem (see [1], [7] and [8]) allows a shortcut in our consistency proof. To this end, we apply a certain algorithm $\mathscr{A}$ to our set of instantiated axioms $T$. The algorithm is as follows: construct val for $T^{\prime}$ and check whether it makes all the axioms in $T \backslash T^{\prime}$ true; if so, return $B$; if not, add 1 to $B$ and repeat as long as it takes to make all the axioms in $T \backslash T^{\prime}$ true. The worst case is that every $\varphi_{i}$ has a counterexample $n_{i}$, compelling the algorithm to possibly run until $B$ is so large that $a_{D}$ surpasses every $\min _{\neg \varphi_{i}}$. The 'while'-loop seems to carry this proof outside PRA, but this is not the case. By Parson's theorem, if $I \Sigma_{1}$ proves that for every $x$ there is a unique value $f(x)$, then the function $f(x)$ is primitive recursive. Equivalently, if $I \Sigma_{1}$ proves that an algorithm (possibly containing 'while'-loops) halts for every input, then the algorithm is actually primitive recursive. The latter is the case for our algorithm $\mathscr{A}$ : it only has to run until $a_{D}>\max _{1 \leq i \leq N} \min _{\neg \varphi_{i}}$, which minorant is a term of $I \Sigma_{1}$. Our direct approach, used above, avoids this advanced conservation result, at the cost of greater length.

In theorem 49 we will significantly upgrade transfer, to the level of several variables not restricted to hypernaturals.

§4. ERNA and NQA $^{ \pm}$vis-à-vis transfer. Recently, Rössler and Jeřábek weakened ERNA's predecessor, the Chuaqui and Suppes system $\mathrm{NQA}^{+}$, into $\mathrm{NQA}^{-}$ by introducing a different axiom schema for external minimization [9]. They also showed that $\mathrm{NQA}^{-}$is more suitable than $\mathrm{NQA}^{+}$for finitistic reasoning in the sense of Tait [12]. We also refer to $\mathrm{NQA}^{\emptyset}$, which is $\mathrm{NQA}^{+}$without minimization axioms.

Most (all) of our ERNA theorems can be proved in $\mathrm{NQA}^{-}\left(\mathrm{NQA}^{+}\right)$without much adaptation; for an example, see after corollary 52. The converse, of course, is not true. While ERNA and $\mathrm{NQA}^{+}$can prove that a standard term $\tau(\vec{x})$ has standard values for standard $\vec{x}, \mathrm{NQA}^{-}$, lacking full external induction, could not.

Our consistency proof of ERNA $+\Pi_{1}$-TRANS is a finite iteration of ERNA's. Likewise, that of $\mathrm{NQA}^{ \pm}+\Pi_{1}$-TRANS would be a finite iteration of that for $\mathrm{NQA}^{ \pm}$. Also, all theorems of ERNA $+\Pi_{1}$-TRANS could be proved in NQA ${ }^{+}+\Pi_{1}$-TRANS and most would also in $\mathrm{NQA}^{-}+\Pi_{1}$-TRANS if the transfer axiom is adapted accordingly. But transfer is too strong for finitism in the sense of Tait. This is evident from the next theorem, to be compared to lemma 4.2 in [9], from which we also adopt the notations.

45. TheOREM. The theory $W K L_{0}$ is interpretable in $\mathrm{NQA}^{\emptyset}+\mathrm{O}-\mathrm{MIN}+\Pi_{1}-\mathrm{TRANS}$.

Proof. In [9], the interpretation of $I \Sigma_{1}$ in $\mathrm{NQA}^{+}$is based on replacing all arithmetical $\Sigma_{1}$-formulas with quantifications relativized to $\mathbb{F N}(x)$, which are in turn replaced by external open formulas, provided by lemma 4.2 of [9]. If this has been done, the $\Sigma_{1}$-induction axioms of $I \Sigma_{1}$ are interpreted as instances of external open induction, which are implied by the schema $\mathrm{O}-\mathrm{MIN}^{\text {st }}$ of $\mathrm{NQA}^{+}$. 
For the interpretation of $I \Sigma_{1}$ in $\mathrm{NQA}^{\emptyset}+\mathrm{O}-\mathrm{MIN}+\Pi_{1}$-TRANS, we start from the same interpretation of arithmetical $\Sigma_{1}$-formulas as quantifications relativized to $\mathbb{F} \mathbb{N}(x)$. Lemma 4.2 in [9] contains the $\mathrm{NQA}^{\emptyset}$-term

$$
m_{\varphi, v_{0}}(\vec{x}):=\left(\mu y \leq v_{0}\left(t_{\varphi(y, \vec{x})}(y, \vec{x})=1\right)\right),
$$

comparable to our ERNA-term $m_{\varphi, \omega}(\vec{x})$ of theorem 58 . Now $\varphi\left(m_{\varphi, v_{0}}(\vec{x}), \vec{x}\right)$ implies $(\exists y)(\mathbb{N}(y) \wedge \varphi(y, \vec{x}))$ and from the latter we obtain $(\exists y)(\mathbb{F} \mathbb{N}(y) \wedge \varphi(y, \vec{x}))$, as $\Sigma_{1}$ transfer is contained in NQA ${ }^{\emptyset}+\mathrm{O}-\mathrm{MIN}+\Pi_{1}$-TRANS. Since all standard numbers are smaller than $v_{0}$, the formula $(\exists y)(\mathbb{F} \mathbb{N}(y) \wedge \varphi(y, \vec{x}))$ implies $\varphi\left(m_{\varphi, v_{0}}(\vec{x}), \vec{x}\right)$ by the definition of $m_{\varphi, v_{0}}$. Thus NQA ${ }^{\emptyset}+\mathrm{O}-\mathrm{MIN}+\Pi_{1}$-TRANS proves the equivalence

$$
(\exists y)(\mathbb{F} \mathbb{N}(y) \wedge \varphi(y, \vec{x})) \leftrightarrow \varphi\left(m_{\varphi, v_{0}}(\vec{x}), \vec{x}\right) .
$$

This equivalence implies that, once all arithmetical $\Sigma_{1}$-formulas have been replaced with quantifications relativized to $\mathbb{F} \mathbb{N}(x)$, the interpreted $\Sigma_{1}$-induction axioms of $I \Sigma_{1}$ are equivalent to instances of internal open induction and hence follow from O-MIN. In section 4.3 of [9] the interpretation of $I \Sigma_{1}$ in $\mathrm{NQA}^{+}$is extended to an interpretation of $W K L_{0}$ in $\mathrm{NQA}^{+}$. Exactly the same technique can be applied here to obtain an interpretation of $W K L_{0}$ in $\mathrm{NQA}^{\emptyset}+\mathrm{O}-\mathrm{MIN}+\Pi_{1}$-TRANS.

§5. Nonstandard machinery available in ERNA. In this section we will gather some basic results for ERNA.

5.1. Fundamental functions of ERNA. For further use we collect here some definable functions, being terms of the language that (provably in ERNA) have the properties of the function. Functions of one hypernatural variable will also be called (hyper) sequences. We will tacitly assume that $\tau(n) \neq \uparrow$ for every hypersequence $\tau(n)$ and every hypernatural $n$.

(i) The identity function $i d(x)=x$ is $\pi_{1,1}$.

(ii) For each constant $\tau$ and each arity $k$, the function

$$
C_{k, \tau}\left(x_{1}, \ldots, x_{k}\right)=\tau,
$$

is $\pi_{k+1, k+1}\left(x_{1}, \ldots, x_{k}, \tau\right)$.

(iii) The hypersequence

$$
r(n)= \begin{cases}0 & \text { if } n=0 \\ 1 & \text { if } n \geq 1\end{cases}
$$

is $\operatorname{rec}_{\sigma \tau}^{k}$ with $k=1, \sigma=0, \tau=C_{2,1}$.

(iv) The function

$$
\zeta(x)= \begin{cases}1 & \text { if } x=0 \\ x & \text { otherwise }\end{cases}
$$

is $1+x-r(\lceil|x|\rceil)$.

(v) The functions

$$
h(x)=\left\{\begin{array}{ll}
1 & \text { if } x>0, \\
0 & \text { otherwise }
\end{array} \quad \text { and } \quad H(x)= \begin{cases}1 & \text { if } x \geq 0, \\
0 & \text { otherwise }\end{cases}\right.
$$

are $\frac{x+|x|}{2 \zeta(x)}$ and $\frac{1}{2}+\frac{\zeta(|x|)}{2 \zeta(x)}$, respectively. 
(vi) For constants $a<b$, the function

$$
1_{(a, b]}(x)= \begin{cases}1 & \text { if } a<x \leq b \\ 0 & \text { otherwise }\end{cases}
$$

is $h(x-a) H(b-x)$. Likewise for the characteristic functions of $[a, b],(a, b)$ and $[a, b)$.

(vii) For constants $a<b$ and terms $\rho(x), \sigma(x), \tau(x)$, the function

$$
d_{a, b, \rho, \sigma, \tau}(x)= \begin{cases}\sigma(x) & \text { if } a<x \leq b \text { and } \rho(x)>0, \\ \tau(x) & \text { otherwise }\end{cases}
$$

is $1_{(a, b]}(x)(h(\rho(x)) \sigma(x)+(1-h(\rho(x))) \tau(x))$. Likewise for $a<x<b$, $a \leq x \leq b$ and $a \leq x<b$ and/or $\rho(x)<0, \rho(x) \leq 0$ and $\rho(x) \geq 0$. Any such construction will be called a definition by cases. The interval may be omitted; if so, $\rho, \sigma, \tau$ in $d_{\rho, \sigma, \tau}$ are allowed to have more than one free variable.

(viii) The function

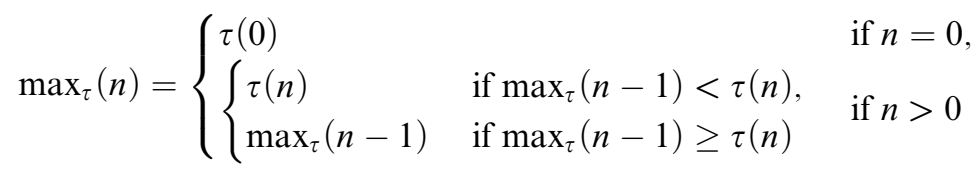

introduced in [10] computes the greatest of all $\tau(m)$ for $m \leq n$.

(ix) The function least $(n):=-\max _{-\tau}(n)$ computes the least of all $\tau(m)$ for $m \leq n$.

(x) The function even $(n):=H(n / 2-\lceil n / 2\rceil)$ decides whether a hypernatural is even or not; likewise for odd $(n)$.

Defining summation and product operators requires the following lemma. Its proof relies on hypernatural induction; this explains why the hypersequence $f(n)$ must be internal.

46. Lemma. Let $f(n)$ be an internal hypersequence, defined for all hypernatural numbers, and not involving $\omega$ or $\min$. If $\|f(n)\| \leq 2_{k}^{n}(k \in \mathbb{N})$, and $g(n)$ is the unary term $\operatorname{rec}_{\sigma \tau}^{k+2}$ obtained from the terms $\sigma=f(0)$ and $\tau(n, x)=f(n)+x$, then

$$
g(n) \text { is defined and }\|g(n)\| \leq 2_{k+2}^{n} \text {. }
$$

Proof. First, it is easily verified by induction that $2 n<2^{n}$ for $n \geq 3$. In particular we have for $n \geq 3$ that $n<2^{n}$ and $n+3<2^{n}$, hence $n(n+3)<2^{2 n}<2^{2^{n}}$. As the inequality $n^{2}+3 n \leq 2^{2^{n}}$ is also valid for $n=0,1,2$, it holds for all $n$.

Next,

$$
2^{2 n}\left(2_{k}^{n}\right)^{n+1} \leq 2_{k+2}^{n}
$$

for all hypernatural numbers $n \geq 0$ and natural $k \geq 1$. For $k=1$ the statement reduces to

$$
2^{n^{2}+3 n} \leq 2^{2^{2^{n}}}
$$

which holds by the last inequality obtained. Now, supposing (8) valid for all hypernatural numbers up to $k$, we estimate $2^{2 n}\left(2_{k+1}^{n}\right)^{n+1}$. The first factor being less 
than the second, the product is at most $\left(2_{k+1}^{n}\right)^{2 n+2}$, i.e., $2^{(2 n+2) 2_{k}^{n}}$. An easy induction shows that $2 n+2 \leq 2^{2 n}$ for $n \geq 1$. Using this in the last estimate, we get

$$
2^{2 n}\left(2_{k+1}^{n}\right)^{n+1} \leq 2^{2^{2 n} 2_{k}^{n}} \leq 2^{2^{2 n}\left(2_{k}^{n}\right)^{n+1}},
$$

also for $n=0$. By the induction hypothesis, the upper bound is at most $2^{2 n} k+2$, i.e., $2_{k+3}^{n}$. This concludes the inductive proof of (8).

It follows from (8) that the statement

$$
g(n) \text { is defined and }\|g(n)\| \leq 2^{2 n}\left(2_{k}^{n}\right)^{n+1}
$$

is stronger than (7). We now prove it by hypernatural induction. For $n=0$ it reduces to $g(0) \neq \uparrow$ and $\|g(0)\| \leq 1$. Since $g(0)=f(0)$ by axiom schema 28, we are left with $\|f(0)\| \leq 2_{k}^{0}$, which is the very assumption for $n=0$. Next, assume that (9) is valid for hypernatural numbers up to $n$. By axiom schema 28 we know that $g(n+1)$ equals $g(n)+f(n+1)$ if this expression is defined and its weight does not exceed $2_{k+2}^{n+1}$. As $g(n)$ and $f(n+1)$ are assumed to be defined, their sum also is. Its weight can be estimated from theorem 24.4, which implies that $\|x+y\| \leq 4\|x\|\|y\|$ if $\|x\| \geq 1$ and $\|y\| \geq 1$. Both $g(n)$ and $f(n+1)$, being defined, have weight $\geq 1$. Hence

$$
\|g(n)+f(n+1)\| \leq 2^{2 n+2}\left(2_{k}^{n}\right)^{n+1} 2_{k}^{n+1}
$$

by the assumptions on the weights of $g(n)$ and $f(n)$. Increasing $\left(2_{k}^{n}\right)^{n+1}$ to $\left(2_{k}^{n+1}\right)^{n+1}$ yields the upper bound $2^{2 n+2}\left(2_{k}^{n+1}\right)^{n+2}$. Therefore (10) implies that

$$
\|g(n+1)\| \leq 2^{2 n+2}\left(2_{k}^{n+1}\right)^{n+2},
$$

which concludes the inductive proof of (9).

47. Lemma. If, in the previous lemma, an estimate $\|f(n)\| \leq 2_{k^{\prime}}^{n}$ is used to obtain a term $g^{\prime}:=\operatorname{rec}_{\sigma \tau}^{k^{\prime}+2}$ instead of $g:=\operatorname{rec}_{\sigma \tau}^{k+2}$, then $g^{\prime}(n)=g(n)$ for all hypernatural numbers $n$.

Proof. As we verified in the previous lemma, $g(n+1)=g(n)+f(n+1)$. Likewise, we have $g^{\prime}(n+1)=g^{\prime}(n)+f(n+1)$. These equations imply a straightforward hypernatural induction.

48. Notation. For an internal term $f(n)$, defined for all hypernatural numbers, and not involving $\omega$ or min, we write

$$
\sum_{0}^{n} f
$$

for the unary term $g(n)$ obtained in lemma 46 . It follows from lemma 47 that this term is independent from the estimate on $\|f\|$. For convenience, we shall also use dummy variables, e.g., $\sum_{i=0}^{n} f(i)$.

To add an extra free variable giving the lower limit, put

$$
\sum_{m}^{n} f= \begin{cases}\sum_{0}^{n} f & \text { if } m=0 \\ \sum_{0}^{n} f-\sum_{0}^{m-1} f & \text { if } 0<m \leq n \\ \uparrow & \text { otherwise }\end{cases}
$$


Starting from a term $f(n, \vec{x})$ with arity $>1$ results in

$$
\sum_{l=m}^{n} f(l, \vec{x})
$$

whose weight is $\leq 2_{k+2}^{\|n, \vec{x}\|}$ if $\|f(n, \vec{x})\| \leq 2_{k}^{\|n, \vec{x}\|}(k \in \mathbb{N})$.

The estimates which theorem 24 gives for $\|x y\|$ are the same as those for $\|x+y\|$. Therefore, all of the preceding can be repeated to yield a product operator $\Pi$ alongside $\sum$.

5.2. Applications of fundamental functions. We now use $\sum$ and $\prod$ to equip ERNA with pairing functions, used to reduce multivariable formulas to singlevariable ones. To encode the couple $(n, m)$ into a unique hypernatural $k$, set

$$
k=2^{n}(2 m+1)-1 .
$$

For the inverse operation, set

$$
m= \begin{cases}k / 2 & \text { if even }(k), \\ \frac{1}{2} \sum_{l=1}^{k}\left(\left(\frac{k+1}{2^{l}}-1\right) \operatorname{odd}\left(\frac{k+1}{2^{l}}\right)\left(1-\prod_{j=0}^{l-1} \text { odd }\left(\frac{k+1}{2^{j}}\right)\right)\right) & \text { otherwise }\end{cases}
$$

and

$$
n=\sum_{l=1}^{k}\left(l \operatorname{odd}\left(\frac{k+1}{2^{l}}\right)\left(1-\prod_{j=0}^{l-1} \operatorname{odd}\left(\frac{k+1}{2^{j}}\right)\right)\right) .
$$

Iterating, we can encode and subsequently decode any finite list of hypernaturals. Thus we can prove the following multivariable form of transfer, not restricted to hypernatural variables.

49. Theorem (Multivariable Tranfer). Assume $\varphi\left(x_{1}, \ldots, x_{k}\right)$ is a quantifier-free formula of $L^{\text {st }}$, not involving min or $\uparrow$. In ERNA $+\Pi_{1}$-TRANS the sentences

$$
\left(\forall^{s t} x_{1}\right) \ldots\left(\forall^{s t} x_{k}\right) \varphi\left(x_{1}, \ldots, x_{k}\right) \text { and }\left(\forall x_{1}\right) \ldots\left(\forall x_{k}\right) \varphi\left(x_{1}, \ldots, x_{k}\right)
$$

are equivalent, and likewise the sentences

$$
\left(\exists x_{1}\right) \ldots\left(\exists x_{k}\right) \varphi\left(x_{1}, \ldots, x_{k}\right) \text { and }\left(\exists^{s t} x_{1}\right) \ldots\left(\exists^{s t} x_{k}\right) \varphi\left(x_{1}, \ldots, x_{k}\right) .
$$

Proof. Because quantifying over hyperrationals amounts to quantifying over couples of hypernaturals, we can restrict ourselves to hypernatural variables. Take $\varphi$ as stated. Let $f\left(n_{1}, \ldots, n_{k}\right)$ be a pairing function encoding $\left(n_{1}, \ldots, n_{k}\right)$ to $n$, and let $f_{1}(n), \ldots, f_{k}(n)$ be its inverse. Then

$$
\left(\forall^{s t} n_{1}\right) \ldots\left(\forall^{s t} n_{k}\right) \varphi\left(n_{1}, \ldots, n_{k}\right)
$$

is equivalent to

$$
\left(\forall^{s t} n\right) \varphi\left(f_{1}(n), \ldots, f_{k}(n)\right) .
$$

All pairing functions being standard, we can transfer to

$$
(\forall n) \varphi\left(f_{1}(n), \ldots, f_{k}(n)\right),
$$

which is equivalent to

$$
\left(\forall n_{1}\right) \ldots\left(\forall n_{k}\right) \varphi\left(n_{1}, \ldots, n_{k}\right) .
$$

Before we can use $\sum$ and $\prod$ to resolve bounded quantifiers, we need the following theorem, interesting in its own right. 
50. THEOREM. For every internal quantifier-free formula $\varphi(\vec{x})$ not involving min or $\uparrow$, ERNA has a function $T_{\varphi}(\vec{x})$ such that

$$
\begin{aligned}
& \varphi(\vec{x}) \text { is true if and only if } T_{\varphi}(\vec{x})=1, \\
& \varphi(\vec{x}) \text { is false if and only if } T_{\varphi}(\vec{x})=0 .
\end{aligned}
$$

Proof. Given such a formula $\varphi(\vec{x})$, resolve every occurrence of $\rightarrow$, leaving only the logical symbols $\wedge, \vee, \neg$. The proof will be completed using induction on the total number $N$ of occurrences of these symbols. If $N=0$, the formula is atomic and, being internal, the form $\tau_{1}(\vec{x}) \approx \tau_{2}(\vec{x})$ is excluded. Three possible types remain to be considered. In defining the corresponding formula $T_{\varphi}$ we use ERNA's function $d_{\rho \sigma \tau}$ defined in (6). For $\tau_{1}(\vec{x}) \leq \tau_{2}(\vec{x})$, take $d_{\tau_{2}-\tau_{1}, 1,0}(\vec{x})$; for $\tau_{1}(\vec{x})=\tau_{2}(\vec{x})$ : $d_{\tau_{2}-\tau_{1}, 1,0}(\vec{x}) d_{\tau_{1}-\tau_{2}, 1,0}(\vec{x})$; finally, for $\mathscr{N}(\tau(\vec{x})): d_{\lceil\tau\rceil-\tau, 1,0}(\vec{x}) d_{\tau-\lceil\tau\rceil, 1,0}(\vec{x}) d_{\tau, 1,0}(\vec{x})$, which expresses that $\lceil\tau(\vec{x})\rceil=\tau(\vec{x})$ and $\tau(\vec{x}) \geq 0$.

Next, assume the theorem holds for all formulas $\psi, \phi, \ldots$ with $N$ occurrences of $\vee, \wedge$ and $\neg$, and consider a formula with one occurrence more. For $\neg \psi(\vec{x})$, take $1-T_{\psi}(\vec{x})$; for $\psi(\vec{x}) \wedge \phi(\vec{x}): T_{\psi}(\vec{x}) T_{\phi}(\vec{x})$, and for $\psi(\vec{x}) \vee \phi(\vec{x}): T_{\psi}(\vec{x})+T_{\phi}(\vec{x})-$ $T_{\psi}(\vec{x}) T_{\phi}(\vec{x})$.

For certain $\vec{x}$, the formula $\varphi(\vec{x})$ may be neither true nor false, for instance $1 / x>0$ for $x=0$. We will tacitly assume that all formulas used have been adapted to exclude such 'critical points'. Here $1 / \zeta(x)>0$ would do.

51. Corollary. For every pair of terms $\sigma(\vec{x}), \tau(\vec{x})$ and every internal quantifierfree formula $\varphi(\vec{x})$ not involving min or $\uparrow$, ERNA has a function

$$
d_{\varphi \sigma \tau}(\vec{x})= \begin{cases}\sigma(\vec{x}) & \text { if } \varphi(\vec{x}), \\ \tau(\vec{x}) & \text { otherwise. }\end{cases}
$$

Proof. Apply definition by cases, as described in $(6)$, to $\rho(\vec{x}):=T_{\varphi}(\vec{x})$. $\quad-$ From now on, 'definition by cases' will include this extension.

52. COROLLARY. For every internal quantifier-free formula $\varphi(n)$ not involving min or $\uparrow$ and every hypernatural $n_{0}$, the internal formula $\left(\forall n \leq n_{0}\right) \varphi(n)$ is equivalent to $\prod_{n=0}^{n_{0}} T_{\varphi}(n)>0$ and, likewise, $\left(\exists n \leq n_{0}\right) \varphi(n)$ is equivalent to $\sum_{n=0}^{n_{0}} T_{\varphi}(n)>0$.

Iterating and combining, we see that, as long as its quantifiers apply to bounded hypernatural variables, every internal formula not involving min or $\uparrow$ can be replaced by an equivalent quantifier-free one.

Essentially, the same result is also proved for the reduced Chuaqui and Suppes system $\mathrm{NQA}^{-}$in lemma 2.4 of [9]. Both proofs can easily be translated from one theory to the other.

Theorem 2 allows us to generalize the preceding corollary as follows.

53. COROLLARY. For every internal quantifier-free formula $\varphi(x)$ not involving $\mathrm{min}$ or $\uparrow$ and every hypernatural $n_{0}$, the sentences $(\exists x)\left(\|x\| \leq n_{0} \wedge \varphi(x)\right)$ and $(\forall x)(\|x\| \leq$ $\left.n_{0} \rightarrow \varphi(x)\right)$ are equivalent to quantifier-free ones.

Next we consider a constructive version of theorem 35. Avoiding the use of $\min _{\varphi}$, it results in functions that can be used in recursion. Along these lines, overflow and underflow can be obtained in theories much weaker than ERNA. 
54. THEOREM. Let $\varphi(n)$ be an internal quantifier-free formula, not involving min or $\uparrow$.

(1) If $\varphi(n)$ holds for every natural $n$, it holds for all hypernatural $n$ up to some infinite hypernatural $\bar{n}$ (overflow).

(2) If $\varphi(n)$ holds for every infinite hypernatural $n$, it holds for all hypernatural $n$ from some natural $\underline{n}$ on (underflow).

Both numbers $\bar{n}$ and $\underline{n}$ are given by explicit ERNA-formulas not involving min.

PROOF.

(1) Suppose $\varphi(n)$ is true for all natural numbers $n$. The hypernatural

$$
\bar{n}:=\sum_{n=1}^{\omega}\left(T_{\varphi}(n) \prod_{k=0}^{n-1} T_{\varphi}(k)\right)
$$

is well-defined in ERNA. As $\varphi(n)$ holds for all natural $n, \bar{n}$ is infinite and its very definition shows that $\varphi(n)$ is true for all $n \leq \bar{n}$.

(2) Likewise,

$$
\underline{n}:=\sum_{n=1}^{\omega}(\omega-n)\left(T_{\neg \varphi}(\omega-n)\left(\prod_{k=0}^{n-1} T_{\varphi}(\omega-k)\right)\right)
$$

is well-defined. If there are hypernatural $n \leq \omega$ for which $\neg \varphi(n), \underline{n}$ is the largest of these. Hence $\underline{n}$ is finite and $\varphi(m)$ holds for all hypernatural $m \geq \underline{n}+1$.

This theorem has some immediate consequences.

55. Corollary. Let $\varphi$ be as in the theorem and assume $n_{0} \in \mathbb{N}$.

(1) If $\varphi(n)$ holds for every natural $n \geq n_{0}$, it holds for all hypernatural $n \geq n_{0}$ up to some infinite hypernatural $\bar{n}$, independent of $n_{0}$.

(2) If $\varphi\left(n_{1}, \ldots, n_{k}\right)$ holds for all natural $n_{1}, \ldots, n_{k}$, it holds for all hypernatural $n_{1}, \ldots, n_{k}$ up to some infinite hypernatural $\bar{n}$.

In both cases the number $\bar{n}$ is given by explicit an ERNA-formula not involving min.

Proof. For (1), take $n_{0}$ as lower limit in (12); for (2), use $k$ summations and $k$ products.

Analogous formulas hold for underflow. Overflow also allows us to prove that the rationals are dense in the finite hyperrationals, being ERNA's version of the 'fundamental theorem of nonstandard analysis'.

56. THEOREM. For every finite a and every natural $n$ there is a rational $b$ such that $|a-b|<\frac{1}{n}$.

Proof. If the stament is false, there exists a finite number $a_{0}$ and a natural $n_{0}$ such that $\left|a_{0}-b\right| \geq \frac{1}{n_{0}}$ for all rational $b$. Then

$$
(\forall b)\left(\|b\| \leq n \rightarrow\left|a_{0}-b\right| \geq \frac{1}{n_{0}}\right)
$$

for all natural $n$. By corollary 53, this formula is equivalent to a quantifier-free formula, and by theorem 54, we can apply overflow. Hence (14) continues to hold 
for $n$ up to some infinite $\omega_{1}$. Defining $\lfloor x\rfloor=-\lceil-x\rceil$, set

$$
\omega_{2}=\left\lfloor\frac{\omega_{1}}{\left\lfloor a_{0}\right\rfloor+1}\right\rfloor
$$

and divide the interval $\left[\left\lfloor a_{0}\right\rfloor,\left\lceil a_{0}\right\rceil\right]$ in subintervals of length $\frac{1}{\omega_{2}} \approx 0$. All points in $\left[\left\lfloor a_{0}\right\rfloor,\left\lceil a_{0}\right\rceil\right]$, in particular $a_{0}$, are infinitely close to a number of the grid. For $m \leq \omega_{2},\left\lfloor a_{0}\right\rfloor+\frac{m}{\omega_{2}}$ is a point of the grid and

$$
\left\|\left\lfloor a_{0}\right\rfloor+\frac{m}{\omega_{2}}\right\|=\left\|\frac{\left\lfloor a_{0}\right\rfloor \omega_{2}+m}{\omega_{2}}\right\| \leq\left\lfloor a_{0}\right\rfloor \omega_{2}+m \leq\left\lfloor a_{0}\right\rfloor \omega_{2}+\omega_{2} \leq \omega_{1} .
$$

Hence all points of the grid have weight less than $\omega_{1}$, contradicting (14) for $n=$ $\omega_{1}$.

57. Notation. We write $(\forall \omega) \varphi(\omega, \vec{x})$ for $(\forall n)(n$ is infinite $\rightarrow \varphi(n, \vec{x}))$. Likewise, $(\exists \omega) \varphi(\omega, \vec{x})$ means $(\exists n)(n$ is infinite $\wedge \varphi(n, \vec{x}))$.

In the following theorem we establish some useful variants of minimization, which will be used in proving theorem 67. Again, they are constructive in avoiding the use of min.

58. THEOREM. Let $M$ be a hypernatural and $\omega_{1}$ an infinite hypernatural. Consider a quantifier-free internal formula $\varphi(n, \vec{x})$ and internal hypersequences $f(n)$ and $g(n)$, none involving min or $\uparrow$.

(1) If there are natural $n$ 's such that $\varphi(n, \vec{x})$, then ERNA has a function $m_{\varphi}(\vec{x})$, with $\left\|m_{\varphi}(\vec{x})\right\| \leq \omega$, which is the least of these.

(2) If there are hypernaturals $n \leq M$ such that $\varphi(n, \vec{x})$, then ERNA has a function $m_{\varphi, M}(\vec{x})$, with $\left\|m_{\varphi, M}(\vec{x})\right\| \leq M$, which is the least of these.

(3) If there are infinite hypernaturals $n \leq \omega_{1}$ such that $\varphi(n, \vec{x})$, then ERNA has a function $m_{\varphi, \omega_{1}}(\vec{x})$ with $\left\|m_{\varphi, \omega_{1}}(\vec{x})\right\| \leq \omega_{1}$, which is the largest of these.

The functions $m_{\varphi}, m_{\varphi, M}$ and $m_{\varphi, \omega_{1}}$ are given by explicit ERNA-functions, not involving $\min$.

Proof. Set

$$
m_{\varphi}(\vec{x})=\sum_{n=1}^{\omega}\left(n T_{\varphi}(n, \vec{x}) \prod_{k=0}^{n-1} T_{\neg \varphi}(k, \vec{x})\right),
$$

yielding a hypernatural which is at most $\omega$. Likewise for $m_{\varphi, M}$. Finally we use 'definition by cases' to obtain $m_{\varphi, \omega_{1}}$, which is equal to

$$
\sum_{n=1}^{\omega_{1}}\left(\left(\omega_{1}-n\right) T_{\varphi}\left(\omega_{1}-n, \vec{x}\right) \prod_{k=0}^{n-1} T_{\neg \varphi}\left(\omega_{1}-k, \vec{x}\right)\right)
$$

if $\neg \varphi\left(\omega_{1}, \vec{x}\right)$, and equal to $\omega_{1}$ otherwise.

The following theorem generalizes overflow to special external formulas.

59. THEOREM. Let $\varphi, f$ and $\omega_{1}$ be as in the previous theorem.

(1) If $f(n)$ is infinite for every $n \in \mathbb{N}$, it continues to be so for all hypernatural $n$ up to some hypernatural number $\omega_{2}$.

(2) If $\left(\forall^{s t} n\right)\left(\exists \omega \leq \omega_{1}\right) \varphi(n, \omega)$, then there is an infinite hypernatural $\omega_{3}$ such that $\left(\forall^{s t} n\right)\left(\exists \omega \geq \omega_{3}\right) \varphi(n, \omega)$. 
Proof. For (1), apply overflow to the formula $f(n)>n$. For $(2)$, let $m_{\varphi, \omega_{1}}(n)$ be the function obtained by applying theorem 58.(3) to $\left(\exists \omega \leq \omega_{1}\right) \varphi(n, \omega)$. Then $m_{\varphi, \omega_{1}}(n)$ is infinite for all $n \in \mathbb{N}$ and by $(1), m_{\varphi, \omega_{1}}(n)$ is infinite for all $n \leq \omega_{2}$ for some infinite $\omega_{2}$. Use 5.1.(ix) to obtain the least of these.

Note that item $(2)$ of the theorem contains $(\forall)(\exists)$, which makes it a $\Pi_{2}$-formula.

§6. Completeness in ERNA $+\Pi_{1}$-TRANS. In this section we will prove an ERNA-version of completeness, to be understood 'up to infinitesimals'. As mentioned before, PRA cannot prove Dedekind completeness (see [6]), and neither can ERNA. We start with Cauchy completeness, which will be used for Dedekind completeness.

\subsection{Hypersequences and Cauchy completeness.}

60. Definition. We say that a hypersequence $\tau(n)$ has the 'standard Cauchy property' if

$$
\left(\forall^{s t} k\right)\left(\exists^{s t} N\right)\left(\forall^{s t} n\right)\left(\forall^{s t} m\right)\left(n, m \geq N \rightarrow|\tau(n)-\tau(m)|<\frac{1}{k+1}\right) .
$$

The first theorem is ERNA's version of a well-known property of Archimedean fields.

61. THEOREM. Let $\tau(n)$ be an internal hypersequence not involving min. Further, let $a$ be a finite constant such that $\tau(n) \leq \tau(n+1) \leq$ a for all natural $n$. Then $\tau(n)$ has the standard Cauchy property.

Proof. If the assertion were false, there would exist some natural $k_{0}$ such that

$$
\left(\forall^{s t} N\right)\left(\exists^{s t} n\right)(\exists m \leq n) \varphi(n, m, N)
$$

where $\varphi(n, m, N)$ stands for

$$
m, n>N \wedge|\tau(n)-\tau(m)| \geq 1 / k_{0}
$$

Apply theorem 58.(1) to $\left(\exists^{s t} n\right)(\exists m \leq n) \varphi(n, m, N)$ and denote the resulting function $m_{\varphi}(N)$ by $f_{1}(N)$. Then apply theorem 58.(2) to $(\exists m \leq n) \varphi(n, m, N)$ and denote the resulting function $m_{\varphi, m}(N, n)$ by $f_{2}(N, n)$. Finally we define $g(n):=f_{2}(h(n-1), h(n))$, where $h(n)$ is the $n$-th iteration of $f_{1}$ at zero. The latter function exists in ERNA because of example 34 .

By construction, all intervals $(\tau(g(l)), \tau(h(l))]$ are disjoint and have length at least $1 / k_{0}$. Therefore, $\sum_{n=2}^{n_{0}+2}|\tau(h(n))-\tau(g(n))|$, with $n_{0}=\left\lceil k_{0}|a-\tau(0)|\right\rceil$, would be larger than $a-\tau(0)$. This clearly is a contradiction, because a finite number of disjoint subintervals cannot have a total length exceeding that of the original interval.

62. Definition. If $a$ is a constant, we say that a hypersequence $\tau(n)$ is 'standard convergent to $a^{\prime}$ if

$$
\left(\forall^{s t} k\right)\left(\exists^{s t} N\right)\left(\forall^{s t} n\right)\left(n \geq N \rightarrow|\tau(n)-a|<\frac{1}{k+1}\right) .
$$

Clearly, the constant $a$ is only unique up to infinitesimals. 
63. THEOREM. Let $\tau(n)$ be an internal hypersequence, not involving $\min$. If $\tau(n)$ has the standard Cauchy property, then there is an infinite hypernatural $m_{0}$ such that all $\tau(m)$ are infinitely close to each other for all infinite $m \leq m_{0}$, and $\tau(n)$ is standard convergent to any of these.

Proof. Choose any natural $k$. By $(15)$ we find a natural $N$ such that

$$
\left(\forall^{s t} m\right)(\forall n \leq m)\left(n, m \geq N \rightarrow|\tau(n)-\tau(m)|<\frac{1}{k+1}\right) .
$$

Corollary 55.(1) yields the infinite hypernatural $\bar{m}(k)$. By theorem 59.(1), it is infinite for all $k \leq \omega_{2}$ for some infinite hypernatural $\omega_{2}$. Let $\omega_{3}$ be the least $\bar{m}(k)$ for $k \leq \omega_{2}$. Then

$$
\left(\forall^{s t} k\right)\left(\exists^{s t} N\right)\left(\forall m \leq \omega_{3}\right)(\forall n \leq m)\left(n, m \geq N \rightarrow|\tau(n)-\tau(m)|<\frac{1}{k+1}\right),
$$

which implies

$$
\left(\forall^{s t} k\right)\left(\exists^{s t} N\right)\left(\forall^{s t} n\right)\left(n \geq N \rightarrow\left|\tau(n)-\tau\left(\omega_{3}\right)\right|<\frac{1}{k+1}\right) .
$$

The internal hypersequence

$$
\tau(n)= \begin{cases}1+\frac{1}{n} & \text { for } n \leq \omega \\ n & \text { otherwise }\end{cases}
$$

shows that there need not be standard convergence to $\tau(n)$ if $n$ is too large an infinite index. In ERNA $+\Pi_{1}$-TRANS we can do considerably better for a hypersequence $\tau(n)$ of $L^{s t}$.

64. THEOREM. Let $\tau(n)$ be a hypersequence of $L^{s t}$, not involving $\min$. If $\tau(n)$ has the standard Cauchy property, then all $\tau(m)$ are infinitely close to each other for all infinite $m$, and $\tau(n)$ is standard convergent to any of these.

Proof. If $\tau(n)$ is as required, we deduce (18) as in the previous theorem. In this formula, fix any $k \in \mathbb{N}$ and find $N \in \mathbb{N}$ such that

$$
\left(\forall^{s t} m\right)\left(\forall^{s t} n\right)\left(n, m \geq N \rightarrow|\tau(n)-\tau(m)|<\frac{1}{k+1}\right) .
$$

In ERNA $+\Pi_{1}$-TRANS, this implies

$$
(\forall m)(\forall n)\left(n, m \geq N \rightarrow|\tau(n)-\tau(m)|<\frac{1}{k+1}\right),
$$

which shows that $\tau(n)$ is standard convergent to $\tau(m)$ for any infinite $m$. Since (20) can be derived for all $k \in \mathbb{N}$, we have $|\tau(n)-\tau(m)|<\frac{1}{k+1}$ for all infinite hypernaturals $n, m$ and $k \in \mathbb{N}$. Hence, infinitely indexed terms differ by infinitesimals.

\subsection{Supremum principles and Dedekind completeness.}

65. Notation. We write $a \ll b$ for $a<b \wedge a \not z b$ and $a \lesssim b$ for $a<b \vee a \approx b$.

We now prove a supremum principle in ERNA $+\Pi_{1}$-TRANS. A preliminary version of it, restricted to particular formulas, is to be found in [4]. In the proof, an indexed term $x_{M}$ means that $\|x\| \leq M$ for some hyperrational. We also use the following property, easily proved by hypernatural induction. 
66. Lemma. Let $\tau(n)$ be an internal term not involving min. If $n_{0}<n_{1}$ are hypernaturals such that $n_{0} \leq n \leq n_{1}-1 \rightarrow \tau(n)=\tau(n+1)$, then $\tau\left(n_{0}\right)=\tau\left(n_{1}\right)$.

67. Theorem (Supremum Principle). Let $b$ be a finite constant and $\varphi(x) a$ quantifier-free formula of $L^{\text {st }}$ not involving $\min$ or $\uparrow$, such that

(i) $\varphi(x)$ holds for no $x>b$,

(ii) $\varphi(x)$ holds for at least one finite $x$.

Then there is a constant $\beta$, given by an explicit ERNA-formula, not involving min, with the following properties:

(iii) $\varphi(x)$ holds for no $x \gg \beta$,

(iv) for every finite $\varepsilon \gg 0$ there are rational $x>\beta-\varepsilon$ such that $\varphi(x)$ holds.

The several constants $\beta$ satisfying these requirements differ by infinitesimals.

Proof. Clearly, we may assume $b$ is rational. Also, transfer guarantees that there is a rational $a^{\prime}$ such that $\varphi\left(a^{\prime}\right)$ holds. Both $b$ and $a^{\prime}$ may be assumed to be positive; if not, consider $\varphi(x+C)$ for a suitable constant $C$. If we set $a=a^{\prime}-1$, $c=b-a>0$ is finite. Then define

$$
\tau(t, n, M)= \begin{cases}t & \text { if } \varphi\left(x_{M}\right) \text { for some } x_{M}>t-\frac{c}{2^{n+1}}, \\ t-\frac{c}{2^{n+1}} & \text { otherwise. }\end{cases}
$$

This definition by cases is justified by corollary 53 , which would allow to eliminate the quantifiers. In order to apply recursion, we choose the rational number $b$ as our term $\sigma$. ERNA's language contains a unary function symbol $\operatorname{rec}_{\sigma \tau}^{1}$.

First we prove that for every hypernatural $n$ and $M$

$$
\varphi\left(x_{M}\right) \rightarrow x_{M} \leq \operatorname{rec}_{\sigma \tau}^{1}(n, M) .
$$

We proceed by hypernatural induction on $n, M$ being fixed. For $n=0,(21)$ reduces to the assumption (i), as $\operatorname{rec}_{\sigma \tau}^{1}(0, M)=\sigma=b$. Now assume that (21) holds for some hypernatural $n$. By axiom schema $28, \operatorname{rec}_{\sigma \tau}^{1}(n+1, M)=\tau\left(\operatorname{rec}_{\sigma \tau}^{1}(n, M), n, M\right)$. If there are $x_{M}>\operatorname{rec}_{\sigma \tau}^{1}(n, M)-c / 2^{n+1}$ such that $\varphi\left(x_{M}\right)$ holds, the definition of $\tau$ implies that $\operatorname{rec}_{\sigma \tau}^{1}(n+1, M)=\operatorname{rec}_{\sigma \tau}^{1}(n, M)$, and

$$
\varphi\left(x_{M}\right) \rightarrow x_{M} \leq \operatorname{rec}_{\sigma \tau}^{1}(n+1, M)
$$

reduces to the induction hypothesis. On the other hand, if there are no such $x$, then $\operatorname{rec}_{\sigma \tau}^{1}(n+1, M)=\operatorname{rec}_{\sigma \tau}^{1}(n, M)-c / 2^{n+1}$ by definition, and (22) also holds.

Next we verify that for every hypernatural $n \geq 1$,

$$
\text { there is an } x_{M}>\operatorname{rec}_{\sigma \tau}^{1}(n, M)-c / 2^{n} \text { such that } \varphi\left(x_{M}\right) \text {. }
$$

Choose any hypernatural $N \geq 1$ and consider the formula

$$
\mathcal{N}(n) \wedge n \leq N-1 \wedge \operatorname{rec}_{\sigma \tau}^{1}(N-n, M) \neq \operatorname{rec}_{\sigma \tau}^{1}(N-n-1, M)-c / 2^{N-n} .
$$

If there are no hypernaturals $n$ satisfying these requirements, then

$$
\operatorname{rec}_{\sigma \tau}^{1}(N-n, M)-c / 2^{N-n}=\operatorname{rec}_{\sigma \tau}^{1}(N-n-1, M)-c / 2^{N-n-1}
$$

for $0 \leq n \leq N-1$, and

$$
\operatorname{rec}_{\sigma \tau}^{1}(N, M)-c / 2^{N}=\operatorname{rec}_{\sigma \tau}^{1}(0, M)-c=a
$$


by lemma 66 . Clearly, $x=a^{\prime}$ satisfies the requirement. If, on the other hand, there are hypernaturals $n$ satisfying (24), let $n_{0}$ be the smallest one, as provided by the second item of theorem 58. Then $n_{0} \leq N-1$ and

$$
\operatorname{rec}_{\sigma \tau}^{1}\left(N-n_{0}, M\right) \neq \operatorname{rec}_{\sigma \tau}^{1}\left(N-n_{0}-1, M\right)-c / 2^{N-n_{0}},
$$

i.e.,

$$
\tau\left(\operatorname{rec}_{\sigma \tau}^{1}\left(N-n_{0}-1, M\right), N-n_{0}-1, M\right) \neq \operatorname{rec}_{\sigma \tau}^{1}\left(N-n_{0}-1, M\right)-c / 2^{N-n_{0}} .
$$

The definition of $\tau(t, n, M)$ implies that

$$
\tau\left(\operatorname{rec}_{\sigma \tau}^{1}\left(N-n_{0}-1, M\right), N-n_{0}-1, M\right)=\operatorname{rec}_{\sigma \tau}^{1}\left(N-n_{0}-1, M\right)
$$

and that there is an

$$
x_{M}>\operatorname{rec}_{\sigma \tau}^{1}\left(N-n_{0}-1, M\right)-c / 2^{N-n_{0}}=\operatorname{rec}_{\sigma \tau}^{1}\left(N-n_{0}, M\right)-c / 2^{N-n_{0}}
$$

satisfying $\varphi\left(x_{M}\right)$. By the leastness of $n_{0}$,

$$
\operatorname{rec}_{\sigma \tau}^{1}(N-n, M)-c / 2^{N-n}=\operatorname{rec}_{\sigma \tau}^{1}(N-n-1, M)-c / 2^{N-n-1}
$$

for $0 \leq n \leq n_{0}-1$. Hence

$$
\operatorname{rec}_{\sigma \tau}^{1}(N, M)-c / 2^{N}=\operatorname{rec}_{\sigma \tau}^{1}\left(N-n_{0}, M\right)-c / 2^{N-n_{0}}
$$

by lemma 66 . Comparing with (26) we see that we have found an

$$
x_{M}>\operatorname{rec}_{\sigma \tau}^{1}(N, M)-c / 2^{N}
$$

such that $\varphi\left(x_{M}\right)$ holds.

Third, for every $n \in \mathbb{N}$, the hypersequence $\operatorname{rec}_{\sigma \tau}^{1}(n, M)$ is an increasing $L^{s t}$ function of $M$, bounded above by $b$. Indeed, since $\sigma=b$ and $\tau(t, n, M) \leq t$, we have $\operatorname{rec}_{\sigma \tau}^{1}(n, M) \leq b$ for all hypernaturals $n$ and $M$. Also, if $\neg \varphi\left(x_{M}\right)$ for all $x_{M}>$ $t-\frac{c}{2^{n+1}}$, and if $\varphi\left(x_{M+1}\right)$ for some $x_{M+1}>t-\frac{c}{2^{n+1}}$, then $\tau(t, n, M)<\tau(t, n, M+1)$; otherwise $\tau(t, n, M)=\tau(t, n, M+1)$. Hence by theorems 61 and 64 , the hypersequence $\operatorname{rec}_{\sigma \tau}^{1}(n, M)$ converges, for any fixed $n \in \mathbb{N}$, to $\operatorname{rec}_{\sigma \tau}^{1}(n, \omega)$. Therefore, $\operatorname{rec}_{\sigma \tau}^{1}\left(n, \omega_{1}\right) \approx \operatorname{rec}_{\sigma \tau}^{1}\left(n, \omega_{2}\right)$ for any $n \in \mathbb{N}$ and infinite hypernaturals $\omega_{1}$ and $\omega_{2}$. Corollary 36 yields an infinite hypernatural $\omega_{3}$ such that $\operatorname{rec}_{\sigma \tau}^{1}\left(n, \omega_{1}\right) \approx \operatorname{rec}_{\sigma \tau}^{1}\left(n, \omega_{2}\right)$ for $n \leq \omega_{3}$.

Fourth, the properties established thus far imply that $\operatorname{rec}_{\sigma \tau}^{1}\left(\omega_{1}, M\right) \approx \operatorname{rec}_{\sigma \tau}^{1}\left(\omega_{2}, M\right)$ for every hypernatural $M$ and infinite hypernaturals $\omega_{1}, \omega_{2}$. Indeed, for any infinite hypernaturals $\omega_{1}$ and $\omega_{2},(23)$ yields $x_{M} \approx \operatorname{rec}_{\sigma \tau}^{1}\left(\omega_{1}, M\right)$ and $x_{M}^{\prime} \approx \operatorname{rec}_{\sigma \tau}^{1}\left(\omega_{2}, M\right)$, both satisfying $\varphi$. By $(21)$, we have $x_{M}, x_{M}^{\prime}<\operatorname{rec}_{\sigma \tau}^{1}\left(\omega_{1}, M\right)$ and likewise for $\omega_{2}$. Hence $\operatorname{rec}_{\sigma \tau}^{1}\left(\omega_{1}, M\right) \approx \operatorname{rec}_{\sigma \tau}^{1}\left(\omega_{2}, M\right)$.

Combining, we conclude that for any four infinite hypernaturals $\omega_{1}, \omega_{2}, \omega_{1}^{\prime}$ and $\omega_{2}^{\prime}$

$$
\operatorname{rec}_{\sigma \tau}^{1}\left(\omega_{1}, \omega_{1}^{\prime}\right) \approx \operatorname{rec}_{\sigma \tau}^{1}\left(\omega_{3}, \omega_{1}^{\prime}\right) \approx \operatorname{rec}_{\sigma \tau}^{1}\left(\omega_{3}, \omega_{2}^{\prime}\right) \approx \operatorname{rec}_{\sigma \tau}^{1}\left(\omega_{2}, \omega_{2}^{\prime}\right)
$$

where $\omega_{3}$ is a small enough infinite hypernatural. Define $\beta:=\operatorname{rec}_{\sigma \tau}^{1}(\omega, \omega)$. From $(21)$ we infer that $(\forall x)(\varphi(x) \rightarrow x \lesssim \beta)$, i.e., (iii). For (iv), fix a finite $\varepsilon \gg 0$. By (23) there is an $x_{0} \approx \beta$ such that $\varphi\left(x_{0}\right)$. If $x_{1}$ is a rational such that $\beta-\varepsilon \gtrsim x_{1} \succsim \beta$, then we have $\left(\exists x>x_{1}\right) \varphi(x)$. By transfer, $\left(\exists^{s t} x>x_{1}\right) \varphi(x)$.

The following version of the supremum principle is provable in ERNA and is not restricted to standard formulas. Uniqueness-up-to-infinitesimals is lost, however. 
68. THEOREM (Internal Supremum Principle). Let $b$ be a finite constant and $\varphi(x)$ an internal quantifier-free formula not involving min or $\uparrow$, such that

(i) $\varphi(x)$ holds for no $x>b$,

(ii) $\varphi(x)$ holds for at least one finite $x$.

Then there is a constant $\beta$, given by an explicit ERNA-formula, not involving min, with the following properties:

(iii) $\varphi(x)$ holds for no rational $x>\beta$,

(iv) for every finite $\varepsilon \gg 0$ there are $x>\beta-\varepsilon$ such that $\varphi(x)$ holds.

PROOF. The proof of the previous theorem can be copied except for the standard convergence of the hypersequence $\operatorname{rec}_{\sigma \tau}^{1}(n, M)$ for fixed $n$ (which can be infinite now). This internal hypersequence is increasing and bounded above by $b$; by theorems 61 and 63, it will converge to $\operatorname{rec}_{\sigma \tau}^{1}\left(n, \omega_{4}\right)$ for all infinite $\omega_{4}$ small enough. It follows that $\operatorname{rec}_{\sigma \tau}^{1}\left(\omega_{1}, \omega_{1}^{\prime}\right) \approx \operatorname{rec}_{\sigma \tau}^{1}\left(\omega_{2}, \omega_{2}^{\prime}\right)$ for infinite $\omega_{1}, \omega_{2}$ and infinite $\omega_{1}^{\prime}, \omega_{2}^{\prime} \leq$ $\omega_{4}$. The properties (iii) and (iv) hold for $\beta:=\operatorname{rec}_{\sigma \tau}^{1}\left(\omega, \omega_{4}\right)$.

Unlike the Internal Supremum Principle 68, the following variant, needing transfer, requires ERNA $+\Pi_{1}$-TRANS. We call it a ' $\Sigma_{1}$-Supremum Principle' because the corresponding formula is $\Sigma_{1}$, whereas in theorem 67 it was quantifier-free.

69. THEOREM $\left(\Sigma_{1}\right.$-Supremum Principle). Let $b$ be a finite constant and $\varphi(x, n) a$ quantifier-free formula of $L^{\text {st }}$ not involving $\min$ or $\uparrow$, such that

(i) $(\exists n) \varphi(x, n)$ holds for no $x>b$,

(ii) $(\exists n) \varphi(x, n)$ holds for at least one finite $x$.

Then there is a constant $\beta$, given by an explicit ERNA-formula, not involving min, with the following properties:

(iii) $(\exists n) \varphi(x, n)$ holds for no $x \gg \beta$,

(iv) for every finite $\varepsilon \gg 0$ there are rational $x>\beta-\varepsilon$ such that $(\exists n) \varphi(x, n)$ holds.

The several constants $\beta$ satisfying these requirements differ by infinitesimals.

Proof. We will apply the previous theorem for $\varphi_{1}(x) \equiv(\exists n \leq \omega) \varphi(x, n)$, which can be treated as a quantifier-free one. Applying theorem 49 to the assumption $(\exists x)(\exists n) \varphi(x, n)$, we have $\left(\exists^{s t} x\right)\left(\exists^{s t} n\right) \varphi(x, n)$, which implies the existence of an $x_{0}$ such that $\varphi_{1}\left(x_{0}\right)$ holds. Assumption (i) implies that $(\forall x>b)(\forall n) \neg \varphi(x, n)$, i.e., $\varphi_{1}(x)$ holds for no $x>b$. By the previous theorem, we obtain a constant $\beta$ such that

(iii)' $(\exists n \leq \omega) \varphi(x, n)$ holds for no rational $x>\beta$,

(iv)' for every finite $\varepsilon \gg 0$ there are $x>\beta-\varepsilon$ such that $(\exists n \leq \omega) \varphi(x, n)$ holds.

The sentence (iii)' implies $\left(\forall^{s t} x>x_{1}\right)\left(\forall^{s t} n\right) \neg \varphi(x, n)$ for all rational $x_{1} \gg \beta$. Transfer yields (iii). For a finite $\varepsilon \gg 0$, (iv)' implies $\left(\exists x>x_{1}\right)(\exists n) \varphi(x, n)$ for any rational $x_{1}$ with $\beta-\varepsilon \succsim x_{1} \succsim \beta$. Transferring, we find (iv). Any other constant $\beta^{\prime}$ satisfying (iii) and (iv) must satisfy $\beta^{\prime} \approx \beta$.

70. ACKNOWLEDGEMENT. The authors thank the referee for drawing their attention to the recent work of Michal Rössler and Emil Jeřábek. They also thank professor Ulrich Kohlenbach (Technische Universität Darmstadt) and professor Andreas Weiermann (University of Ghent) for their valuable advice. 


\section{REFERENCES}

[1] Samuel Buss, Proof theory, Elsevier, 1998.

[2] Rolando Chuaqui and Patrick Suppes, Free-variable axiomatic foundations of infinitesimal analysis: a fragment with finitary consistency proof, this JoURNAL, vol. 60 (1995), pp. 122-159.

[3] Petr HádeK and Pavel Pudlák, Metamathematics of first-order arithmetic, Springer, 1998.

[4] CHRIS IMPENS and SAM SANDERS, ERNA at work, The strength of nonstandard analysis (Imme van den Berg and Vitor Neves, editors), Springer, 2007, pp. 64-75.

[5] Vladimir KANOVEI and Michael REEKEN, Nonstandard analysis, axiomatically, Springer, 2004.

[6] Ulrich Kohlenbach, Things that can and things that can't be done in PRA, Annals of Pure and Applied Logic, vol. 102 (2000), pp. 223-245.

[7] Charles Parsons, On a number-theoretic choice scheme and its relation to induction, Intuitionism and proof theory (J. Myhill, A. Kino, and R. E. Vesley, editors), North-Holland, 1970, pp. 459-473.

[8] — On n-quantifier induction, this JournaL, vol. 37 (1972), pp. 466-482.

[9] MiCHAL RÖSSLER and EMIL JEŘÁBEK, Fragment of nonstandard analysis with a finitary consistency proof, The Bulletin of Symbolic Logic, vol. 13 (2007), pp. 54-70.

[10] RiCHARD SOMMER and PATRICK SUPPES, Finite models of elementary recursive nonstandard analysis, Notas de la Sociedad Matemática de Chile, vol. 15 (1996), pp. 73-95.

[11] Keith D. Stroyan and Willem A. J. Luxemburg, Introduction to the theory of infinitesimals, Academic Press, 1976.

[12] William W. Tait, Finitism, The Journal of Philosophy, vol. 78 (1981), pp. 524-564.

UNIVERSITY OF GHENT

DEPARTMENT OF PURE MATHEMATICS AND COMPUTER ALGEBRA

GALGLAAN 2

B-9000 GENT, BELGIUM

E-mail: ci@cage.ugent.be

$U R L:$ http://cage.ugent.be/ $\sim_{\text {ci }}$

E-mail: sasander@cage.ugent.be

$U R L:$ http://cage.ugent.be $/ \sim_{\text {sasander }}$ 